\title{
A FERROELECTRIC CAPACITOR (FECAP) BASED UNRELEASED RESONATOR
}

\author{
Yanbo He $e^{l}$, Bichoy Bahr ${ }^{2}$ and Dana Weinstein ${ }^{1}$ \\ ${ }^{1}$ Purdue University, West Lafayette, IN, USA \\ ${ }^{2}$ Kilby Labs, Texas Instruments, Dallas, TX, USA
}

\begin{abstract}
We present the first $\mathrm{Pb}(\mathrm{Zr}, \mathrm{Ti}) \mathrm{O}_{3}$ ( $\mathrm{PZT}$ )-transduced unreleased $\mathrm{RF}$ MEMS resonator embedded seamlessly in CMOS. The unreleased resonators were realized by using Texas Instruments' Ferroelectric RAM (FeRAM) 130nm technology [1]. A new method of acoustic waveguiding within the CMOS stack was used to confine the resonance mode and an array of ferroelectric capacitors (FeCAPs) inherent to the technology was used as driving and sensing transducers. A resonance frequency of 722 $\mathrm{MHz}$ is observed with quality factor $\mathrm{Q}$ of 656 and electromechanical coupling coefficient $\mathrm{k}_{\mathrm{eff}}{ }^{2}$ of $0.35 \%$. This corresponds to $\mathrm{f} \cdot \mathrm{Q}$ of $4.7 \times 10^{11}$, comparable to state-of-the-art PZT resonators [2]. These high Q, small footprint resonators offer a CMOS-integrated RF building block with no post-processing or costly packaging.
\end{abstract}

\section{INTRODUCTION}

Over the past decades, the boom in development of RF communications has enabled a range of applications including high bandwidth $5 \mathrm{G}$ radio, wearable radars, mm-wave imaging, and beyond. The demanding performance of these systems alongside requirements for miniaturization, lower power consumption, and lower cost have pushed the limits on what conventional technology can currently achieve [3]. With high-Q and small footprint, CMOS-MEMS resonators can provide ideal $\mathrm{RF}$ building blocks for filters, low phase noise clocks, and sensors.

The authors have previously demonstrated high- $Q$, unreleased resonators termed Resonant Body Transistors (RBTs) [4] standard CMOS technology at frequencies ranging from $3 \mathrm{GHz}$ [5] to $32 \mathrm{GHz}$ [6]. These resonators leverage the MOS capacitors inherent to the process for electrostatic actuation to drive acoustic resonance in a solid-state acoustic 'cavity' defined using the CMOS layers. While the $f$.Q products of these devices are record breaking, their insertion loss (IL) is limited by the fundamental limits of electrostatic transduction, which provides limited driving force. This is particularly evident in the case of planar CMOS technology (e.g. $32 \mathrm{~nm} \mathrm{SOI}$ ), where the electromechanical transconductance for the $3 \mathrm{GHz}$ resonators is on the order of $100 \mathrm{nS}$. The correspondingly high IL of such a device makes oscillator and filter design very challenging. While FinFET technology has proven an effective way to maximize output signal in spite of electrostatic drive [6], ferroelectric memorybased CMOS technologies offer higher transducer coupling to minimize IL by leveraging piezoelectric materials introduced in the back-end-of-line (BEOL) process. Higher coupling $\mathrm{k}^{2}$ also translates to larger bandwidth filters and higher frequency tolerance to fabrication variations.

In this work, we focus on piezoelectric resonators designed in Texas Instruments' Ferroelectric RAM (FeRAM) process. TI E035 technology contains ferroelectric PZT capacitors integrated within the metal layers of the CMOS stack, utilizing the hysteresis characteristics for memory devices (Fig. 1) [1].

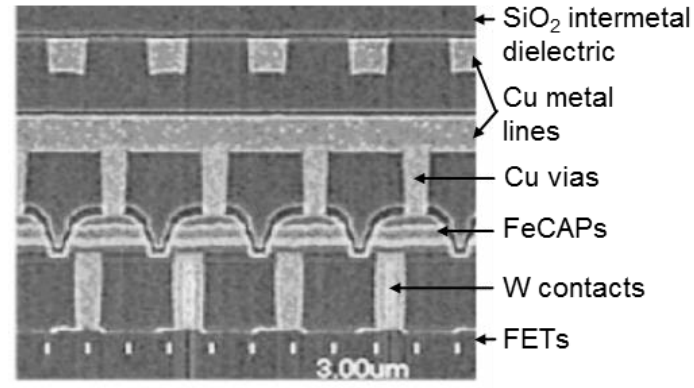

Figure 1: Cross sectional SEM of TI E035 ferroelectric RAM (FeRAM) CMOS technology [1], showing ferroelectric PZTbased capacitors (FeCAPS) within the metal stack.

These devices are the first implementation of ferroelectric capacitor (FeCAP) based unreleased resonators.

This work differs from previously reported devices [4-6] in 3 important ways: (1) The RBTs rely on electrostatic transduction using MOSCAPs with fundamentally lower $\mathrm{keff}^{2}$ than the PZTbased resonators in this work. (2) RBTs use transistor sensing to detect the resonance, while the FeCAP resonator is entirely passive. (3) The FeCAP resonator only requires the first metal layer for vertical acoustic confinement, as opposed to a Phononic Crystal composed of all metal layers above the resonance cavity.

\section{SIMULATION AND DESIGN}

The side view schematic of the resonator is plotted in Fig. 2, showing an array of trapezoidal FeCAPs each spanning $1.75 \mathrm{um}$ in length, connected to Copper strips by Tungsten vias. The resonator consists of 10 driving transducers and 10 sensing transducers alternating with $2 \mathrm{um}$ periodicity to form the resonant cavity. To achieve high-Q resonators, it is essential to laterally confine the energy into the resonance cavities while maximizing stress in the vertical direction at the PZT layer within the FeCAP. This is achieved using acoustic waveguiding [4] within the CMOS stack.

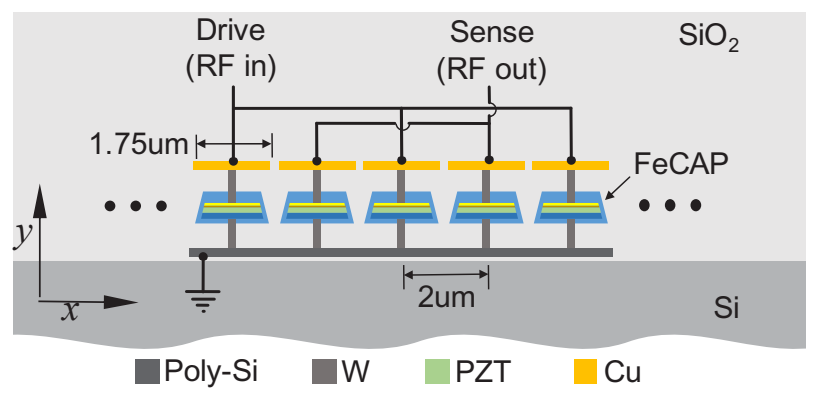

Figure 2: Cross section schematic of FeCAP based resonator (not to scale). A set of 10 drive and 10 sense transducers alternate with 2 um periodicity to form an acoustic waveguide in the CMOS $B E O L$. The resonance cavity is confined laterally with additional dummy FeCAP unit cells on either side of the transducer array. 
To achieve acoustic waveguiding in CMOS, one must first determine the dispersion relations of the stack assuming lateral (x-direction) translational symmetry. In other words, assuming a repeating structure, we want to find modes that are confined to propagate only in the plane of the wafer. In this case, the periodic structure is the single FeCAP with a $1.75 \mathrm{um}$ long $\mathrm{Cu}$ strip above it in the BEOL. This unit cell is $a=2 \mu \mathrm{m}$ in length. The boundaries are defined as Floquet periodic boundary conditions with $k_{x} \pi / a$ periodicity. This gives the width of a single period as $\pi / a$. This corresponded to the lateral width of the first irreducible Brillouin zone in the reciprocal lattice. The simulation was performed in COMSOL Multiphysics. By searching the eigenmodes along the first irreducible Brillouin zone, the dispersion relations can be mapped as shown in Fig. 3(a).

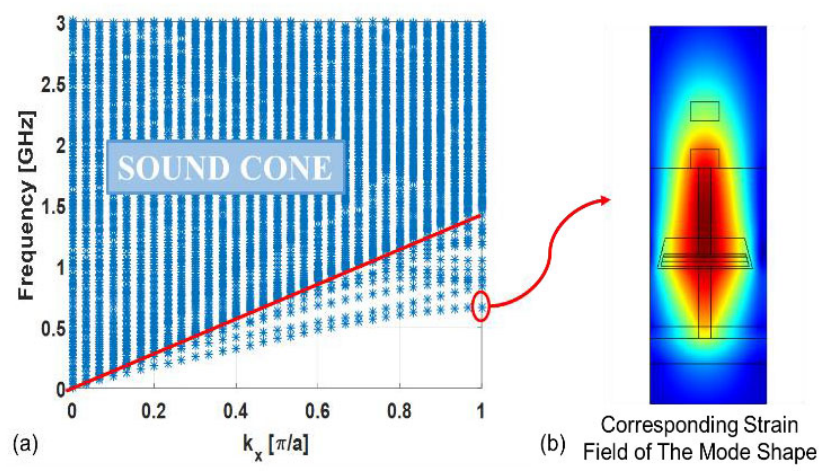

Figure 3: (a) Dispersion relation showing the vertically confined mode. Modes in the sound cone are free to propagate into the bulk Si substrate and cannot be confined. (b) Corresponding localized mode shape of the unit cell FeCAP.

Each point in the dispersion relation (Fig. 3(a)) corresponds to an eigenmode of the periodic FeCAP structure. This dispersion relation can be divided into two regions separated by a sound-line (red) represented by $\omega=c \cdot k$, where $\mathrm{c}$ is the shear acoustic velocity in the Si substrate. Above the sound-line is a region where all the modes are free to propagate, referred to as the sound cone. Below the sound line exist several discrete modes with sufficiently low acoustic velocity that they are prohibited from propagation in the bulk and are therefore confined in the BEOL region of the CMOS chip.

It was previously shown [4] that driving near $k_{x}=\pi / a$ is beneficial for reducing scattering to the sound cone, enabling larger quality factor. Fig. 3(b) shows a vertically confined mode at $700 \mathrm{MHz}$ where the strain is well contained in the FeCAP, W via as well as the $\mathrm{Cu}$ metal. In practice, the periodic transduction across $20 \mathrm{FeCAPs}$ reinforces the targeted mode with $k_{\mathrm{x}}=\pi / a$ wavenumber. Meanwhile, to laterally confine the waveguided mode, additional dummy FeCAP unit cells are placed on either side of the array. These elements are spaced with the same period as the FeCAP transducers in the resonant cavity.

\section{EXPERIMENTAL RESULTS AND ANALYSIS}

The aforementioned devices were fabricated using Texas Instruments' ferroelectric RAM (FeRAM) 130nm technology. Fig. 4 shows a cross-sectional SEM of the device, revealing the periodic FeCAP unit cells above the bulk $\mathrm{Si}$ substrate and surrounded by $\mathrm{SiO}_{2}$ in the BEOL.

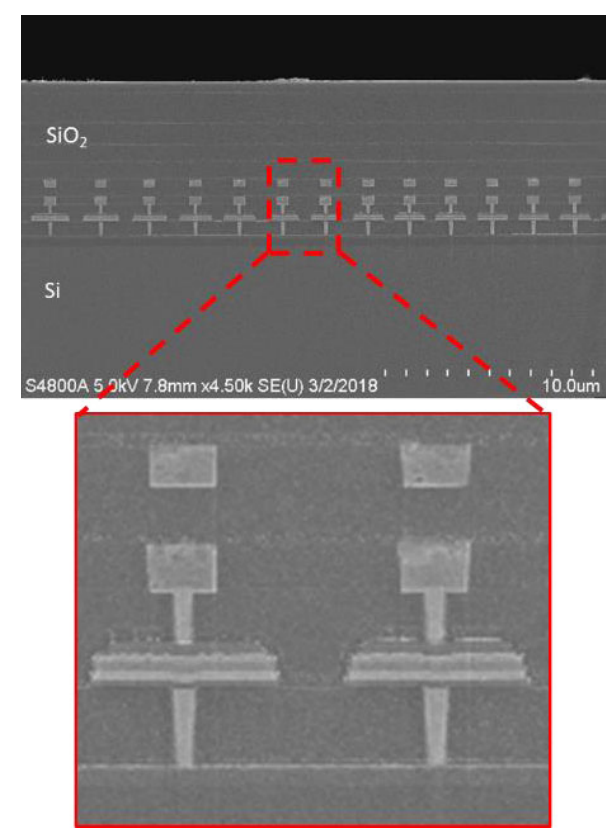

Figure 4: SEM of the waveguided CMOS resonator, showing periodic transducers (alternating drive and sense). Zoom in highlights details of the FeCAPs, with a thin layer of PZT sandwiched between mutli-layered electrodes [1].

The ferroelectric properties of the FeCAP were first characterized to investigate PZT film quality. It should be noted that the film is optimized by the foundry for FE properties for memory and not specifically for piezoelectric coupling. Shown in Fig. 5 is the measured hysteresis of PZT FeCAP polarization.

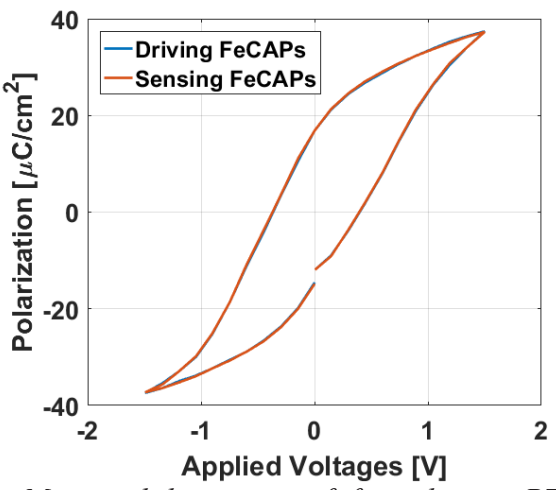

Figure 5: Measured hysteresis of ferroelectric PZT FeCAP polarization. For efficient piezoelectric transduction, a polarization voltage $V_{P}$ between $-1.5 \mathrm{~V}$ and $1.5 \mathrm{~V}$ is applied across the device.

The operational polarization voltage $V_{P}$ is between $-1.5 \mathrm{~V}$ and $1.5 \mathrm{~V}$. For a total FeCAP area of $98 \mathrm{um}^{2}$, the maximum polarization obtained is $38 \mathrm{uC} / \mathrm{cm}^{2}$. With this in mind, the 2-port RF measurements were then performed in ambient temperature and pressure using a Keysight PNA N5225A. Two Keithley 2400 s were connected to the PNA to set the DC bias. The experimental setup was shown in Fig. 6. 


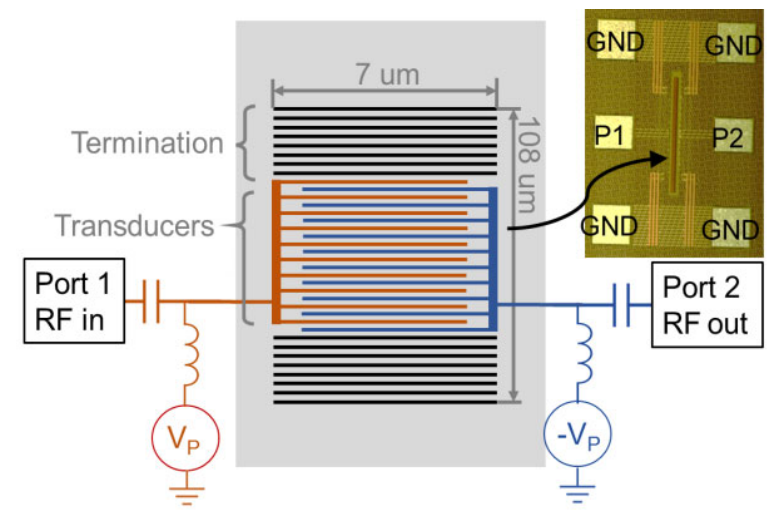

Figure 6: Experimental setup showing top view schematic of resonator. Drive (red) and sense (blue) transducers alternate within the resonator to for the cavity. The acoustic mode is confined laterally using an array of electrically floating FeCAPs (black). Device footprint measures $108 \times 7 \mathrm{um}$.

Driving transducers were connected to Port 1 of the PNA and were biased at $1.5 \mathrm{~V}$ whereas the sensing transducers connected to Port 2 and were biased at $-1.5 \mathrm{~V}$. The resonators were biased in this way to enforce the excitation of the desired mode at the Brillouin zone edge $(k=\pi / \mathrm{a})$. The RF power was set as $-15 \mathrm{dBm}$ with an IF bandwidth (IFBW) of $500 \mathrm{~Hz}$ with 30 -point sweep averaging.

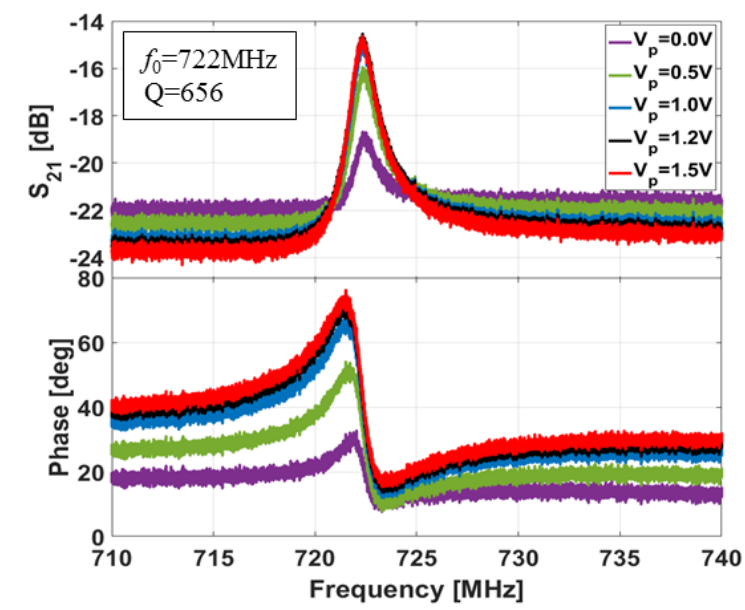

Figure 7 Measured frequency response of FeCAP. Device S21 has been de-embedded from pad/routing parasitics using on-chip Open and Short structures.

Fig. 7 has shown the measured frequency response of the proposed FeCAP resonator. A resonance was observed at 722 $\mathrm{MHz}$. With the increase of polarization voltages, the amplitude of $\mathrm{S}_{21}$ increases correspondingly. Table 1 outlines the key specification as a function of $V_{P}$.

As shown in Table 1, $Q$ increases with respect to the increasing $V_{P}$ reaching 656 at the maximum polarization voltage of $1.5 \mathrm{~V}$. This corresponds to an $f: Q$ of $4.74 \times 10^{11}$. FeCAP transducer capacitance $\mathrm{C}_{0}$ and electromechanical coupling coefficient $\mathrm{keff}^{2}$ were fitted in Advanced Design System (ADS) to a Modified Butterworth-Van-Dyke model. As demonstrated in Table 1, with the increase of polarization voltage, the corresponding $\mathrm{C}_{0}$ decreases from $21.36 \mathrm{pF}$ to $14.06 \mathrm{pF}$. The obtained $\mathrm{keff}^{2}$ is $0.35 \%$ is lower than typical PZT due to film optimization for ferroelectric hysteresis (memory function) rather than piezoelectric coupling. The maximum Figure of Merit (FOM) $\mathrm{k}^{2} \mathrm{Q}$ is 2.3. The demonstrated devices achieve comparable $f \cdot Q$ relative to state-of-the-art PZT resonators in a monolithic CMOS platform.

\begin{tabular}{|c|c|c|c|c|c|}
\hline $\begin{array}{l}V_{P} \\
(\mathrm{~V})\end{array}$ & $Q$ & $\begin{array}{r}C_{0} \\
(\mathrm{pF})\end{array}$ & $k_{e f f}{ }^{2}$ & $\underset{\left(\times 10^{11}\right)}{f \cdot Q}$ & $k^{2} \cdot Q$ \\
\hline 0.0 & 122 & 21.36 & \multirow{5}{*}{$0.35 \%$} & 0.88 & 0.43 \\
\hline 0.5 & 451 & 17.81 & & 3.26 & 1.58 \\
\hline 1.0 & 557 & 14.82 & & 4.02 & 1.95 \\
\hline 1.2 & 602 & 13.86 & & 4.35 & 2.12 \\
\hline 1.5 & 656 & 14.06 & & 4.74 & 2.30 \\
\hline
\end{tabular}

Table 1. Key performance of the FeCAP resonator with respect to the increasing driving voltages, with performance improving up to the maximum technology voltage $V_{P}=1.5 \mathrm{~V}$.

Temperature stability measurement were also performed on on the FeCAP resonators. Frequency response was obtained between room temperature and $90^{\circ} \mathrm{C}$. The extracted Temperature Coefficient of Frequency (TCF) was $-58.1 \pm 4.6 \mathrm{ppm} /{ }^{\circ} \mathrm{C}$. This temperature sensitivity matches well to predicted values based on the Temperature Coefficient of Young's Modulus ( TCE = $\Delta E / \Delta T$ ) of the constituent materials in the CMOS BEOL. Both PZT and $\mathrm{SiO}_{2}$ have positive TCE while $\mathrm{W}$ via has negative TCE. In the meantime, most of the strain energy of the resonance mode is localized in the W via (as shown in Fig. 3(b)). Future design may consider BEOL metal geometry for passive temperature compensation.

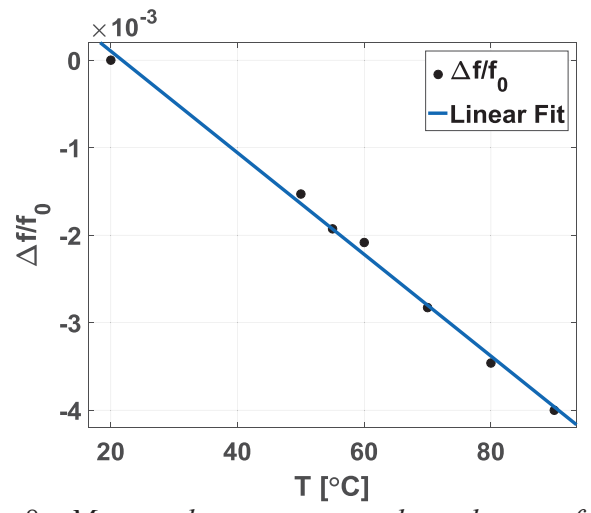

Figure 8: Measured temperature dependence of resonance frequency. A Temperature Coefficient of Frequency $T C F=\Delta f / f 0 / \Delta T$ of $-58 \mathrm{ppm} / \mathrm{K}$ is extracted, owing primarily to energy localization in the $W$ vias.

Resonator phase noise, we are using the residue phase noise method [11] with schematic shown in Fig. 9.

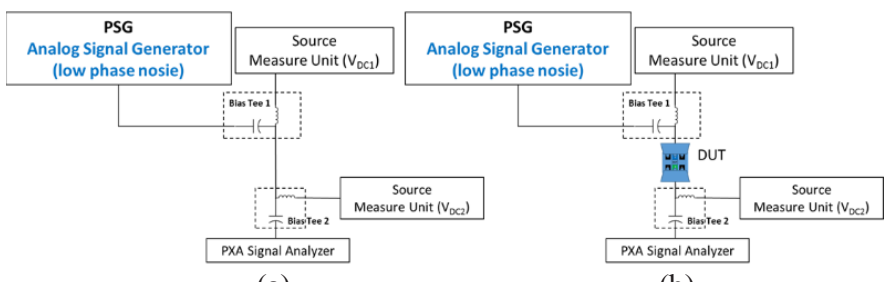

(a)

(b)

Figure 9: Schematic of the residue phase noise measurement. 


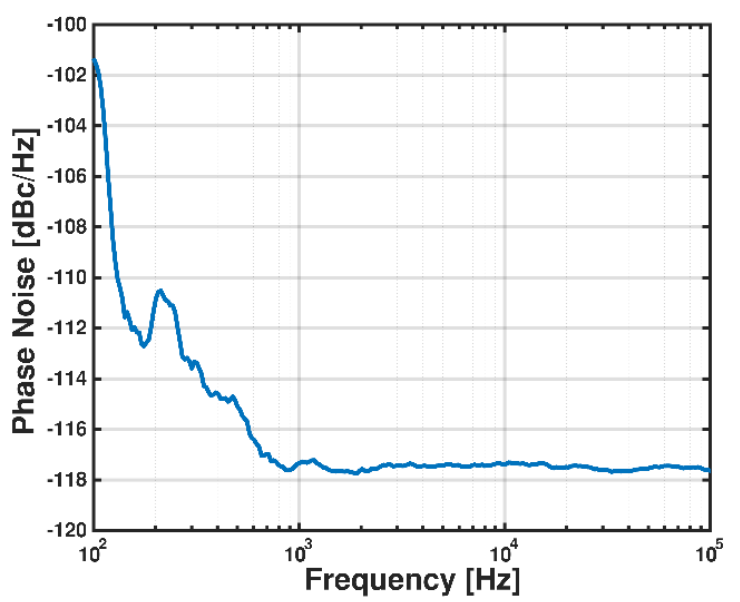

Figure 10: Measured phase noise of the $722 \mathrm{MHz} F e C A P$ resonator, exhibiting $-118 \mathrm{dBc} / \mathrm{Hz}$ at $1 \mathrm{kHz}$ offset from carrier.

The phase noise was measured without the device under test (DUT) (Fig. 9(a)) and with DUT (Fig. 9(b)). The RF signal generator was set in a low phase noise mode. The phase noise of the resonator is then obtained by deembedding the measurement noise from the resonator by:

$$
10^{\frac{\mathcal{L}_{\text {component }}}{10}}=10^{\frac{\mathcal{L}_{\text {with DUT }}}{10}}-10^{\frac{\mathcal{L}_{\text {without DUT }}}{10}} .
$$

The resulting phase noise of the $722 \mathrm{MHz}$ FeCAP resonator is plotted in Fig. 10. A broadband noise floor of $-118 \mathrm{dBc} / \mathrm{Hz}$ is measured at $1 \mathrm{kHz}$ offset.

\section{CONCLUSION}

This work presents the first demonstration of a PZT-transduced RF MEMS resonator embedded seamlessly in CMOS, leveraging Texas Instrument's FeRAM 130nm technology. The proposed resonator exhibits resonance frequency of $722 \mathrm{MHz}$ and $\mathrm{Q}$ of 656 , alongside an electromechanical coupling coefficient $\mathrm{keff}^{2}$ of $0.35 \%$. This corresponds to comparable $f \cdot Q$ of $4.7 \times 10^{11}$ in comparison to state-of-the-art PZT resonators. Table 2 provides a comparison to other IC-integrated and piezoelectric resonators, including previously reported Resonant Body Transistors in GaN MMIC technology. As evident from the table, this work shows higher $\mathrm{keff}^{2}$ than the GaN MMIC-integrated resonators primarily due to limits of the material [7-8]. However, this work achieved comparable $f$.Q products with most of previous PZT resonators [2], [10], but with the added benefit of direct CMOS integration.

\section{ACKNOWLEDGEMENT}

The authors thank Texas Instruments for in-depth design and layout discussions and SEMs, as well as for fabrication of the devices presented here. This work was funded by the DARPA MTO UPSIDE program.

\begin{tabular}{|c|l|l|c|c|c|c|c|c|}
\hline Reference & \multicolumn{1}{|c|}{ Type of device } & IC & $\mathrm{C}_{0}$ & $\mathrm{keff}^{2}$ & $f(\mathrm{GHz})$ & $\mathrm{Q}$ & $f \cdot \mathrm{Q}\left(\times 10^{11}\right)$ & $\mathrm{k}^{2} \cdot \mathrm{Q}$ \\
\hline$[7]$ & GaN Lamb mode RBT & MMIC integrated & $17 \mathrm{fF}$ & $0.17 \%$ & 1.011 & 5509 & 55.70 & 9.37 \\
\hline$[8]$ & GaN thickness mode RBT & MMIC integrated & $1.59 \mathrm{pF}$ & $0.08 \%$ & 4.230 & 250 & 10.58 & 0.20 \\
\hline$[2]$ & PZT FBAR & Not compatible & - & $52.7 \%$ & 3.724 & 114 & 4.245 & 60.08 \\
\hline$[9]$ & PZT contour mode resonator & Not compatible & $1.55 \mathrm{pF}$ & $0.05 \%$ & 0.011 & 6575 & 0.704 & 3.29 \\
\hline$[10]$ & AlN contour mode resonator & CMOS compatible & $1.25 \mathrm{pF}$ & $0.54 \%$ & 0.223 & 4300 & 9.589 & 23.22 \\
\hline This Work & FeRAM CMOS resonators & CMOS integrated & $14.06 \mathrm{pF}$ & $0.35 \%$ & 0.722 & 656 & 4.736 & 2.30 \\
\hline
\end{tabular}

Table 2. Comparison of the key performance of the FeCAP resonators with previously reported IC-compatible and PZT devices.

\section{REFERENCE}

[1] K. Udayakumaret, et. al. "Manufacturable High-Density 8 Mbit One Transistor-One Capacitor Embedded Ferroelectric Random Access Memory." Japanese Journal of Applied Physics 47.4S (2008): 2710

[2] B. Harmeet, and G. Piazza, eds. Piezoelectric MEMS Resonators. Springer, 2017 (Chapter 2).

[3] B. Bhar. Monolithically integrated MEMS resonators and oscillators in standard IC technology. Diss. Massachusetts Institute of Technology, 2016.

[4] D. Weinstein, and S. Bhave. "The resonant body transistor." Nano letters 10.4 (2010): 1234-1237.

[5] B. Bahr, D. Weinstein, "Vertical Acoustic Confinement for High-Q Fully-Differential CMOS-RBTs," Hilton Head 2016.

[6] B. Bahr, Y. He, et al., "32GHz Resonant-Fin Transistors in 14nm FinFET Technology" to be presented at ISSCC 2018.

[7] L. Popa and D. Weinstein. "L-band Lamb mode resonators in gallium nitride MMIC technology." Frequency Control Symposium (FCS), 2014 IEEE International. IEEE, 2014.

[8] A. Ansari, and M. Rais-Zadeh. "A thickness-mode $\mathrm{AlGaN} / \mathrm{GaN}$ resonant body high electron mobility transistor." IEEE Transactions on Electron Devices 61.4 (2014): 1006-1013. [9] J. Pulskamp, et al. "Ferroelectric PZT MEMS HF/VHF resonators/filters." Frequency Control Symposium (IFCS), 2016 IEEE International. IEEE, 2016.
[10] G. Piazza, J. S. Philip, and P. Albert. "Piezoelectric aluminum nitride vibrating contour-mode MEMS resonators." Journal of Microelectromechanical systems 15.6 (2006): 14061418.

[11] J. Breitbarth, and K. Joe. "Additive (residual) phase noise measurement of amplifiers, frequency dividers and frequency multipliers." Microwave Journal 51.6 (2008): 66.

*Y.He, he355@purdue.edu 